\title{
EL CONSENTIMIENTO INFORMADO EN ODONTÓLOGOS RESIDENTES DE LA FACULTAD DE ODONTOLOGÍA DE LA UNIVERSIDAD NACIONAL DE LA PLATA
}

\author{
Ricardo Miguel ${ }^{*}$ \\ Martín Zemel * $^{* *}$
}

Resumen: Este trabajo se fundamenta en la necesidad de obtener datos sobre la práctica del consentimiento informado realizado por odontólogos. Los datos pertenecen al Programa de Residencias Odontológicas Universitarias (ROU) de la Facultad de Odontología de la Universidad Nacional de La Plata (UNLP). La investigación tiene como intención conocer diferentes aspectos respecto a las fortalezas y debilidades de su utilización actual.

A partir de los datos, se puede concluir que, a pesar de que los conocimientos teóricos sobre la práctica del consentimiento informado son escasos, existe una tendencia significativa a destinar tiempo en la consulta dental para su empleo, especialmente en la nueva generación. Se considera imperativo incluir en la malla curricular y en los programas académicos odontológicos principios éticos y legales en relación con el proceso de consentimiento informado.

Palabras clave: consentimiento informado, odontología, posgrado

\section{INFORMED CONSENT IN ODONTOLOGY RESIDENTS AT THE DENTISTRY FACULTY, LA PLATA'S NATIONAL UNIVERSITY}

\begin{abstract}
This work is based upon the need to obtain data on the practice of informed consent carried out by dentists. Data was generated from the University Residence Program at the Dentistry Faculty, L.P.N.U., Argentina. The investigation's goal is to learn about different aspects regarding strengths and weaknesses of informed consent practice.

One of the findings was that theoretical knowledge on the practice of informed consent is scarce, but the new generation of dentists is dedicating more time to it. It is imperative to include ethical and legal principles related to informed consent in all dental academic curricula.
\end{abstract}

Key words: informed consent, dentistry, graduate degrees

\section{O CONSENTIMENTO INFORMADO EM ODONTÓLOGOS RESIDENTES DA FACULDADE DE ODONTOLOGIA DA UNIVERSIDADE NACIONAL DE LA PLATA}

Resumo: Este trabalho fundamenta a necessidade de se obter dados sobre a prática do consentimento informado realizado por odontólogos. Os dados pertencem ao Programa de Residencias Odontológicas Universitárias (ROU) da Faculdade de Odontologia da Universidade Nacional de la Plata (UNLP). A pesquisa tem como objetivo conhecer os diferentes aspectos, a respeito das forças e fraquezas de sua utilzação atual. A partir dos dados, se pode concluir que, apesar de serem escassos os conhecimentos teóricos sobre a prática do consentimento informado, existe uma tendencia significativa a destinar tempo na consulta dental para seu uso, especialmente na nova geração. Considera-se um imperativo incluir no curriculo de formação profissional e nos programas acadêmicos odontológicos, princípios éticos e legais relacionados com o processo do consentimento informado.

Palavras chave: consentimento informado, odontologia, pós-graduação

\footnotetext{
* Profesor en la Facultad de Odontología de la Universidad Nacional de La Plata, Argentina

** Odontólogo, Universidad Nacional de La Plata, Argentina

Correspondencia:mzemel@uolsinectis.com.ar
} 


\section{Introducción}

\section{El documento de consentimiento informado desde la perspectiva bioética}

En los últimos años, los cambios producidos en el área de la salud y, en particular, en la odontología, motivaron el nacimiento de un nuevo paradigma en la relación odontólogopaciente. El nuevo modelo imperante exige al profesional informar a su paciente todo lo concerniente a su salud bucal. Por tal motivo, surge el documento de consentimiento informado como instrumento escrito que pone de manifiesto las voluntades expresadas, desplegando una nueva relación de tipo contractual: el derecho del paciente a la elección responsable y el respeto a la propia libertad sobre su cuerpo y su salud. De esta forma, el derecho a la información aparece como una manifestación concreta del derecho a la protección de la salud y éste, a su vez, como uno de los fundamentales derechos de la persona humana.

El consentimiento informado, definido por Beauchamp \& Childress como la autorización para iniciar un plan de tratamiento, debiera darse en un lenguaje adecuado al paciente, de manera que pueda comprender los beneficios que obtendrá, las obligaciones que asume y los riesgos que corre(1). En caso de que el tratamiento sugerido no fuera aceptado, es necesario dejar constancia de ello. Así quedará en claro tanto el proceso que culmina con la aceptación como la eventual negativa(2).

El concepto de informar las decisiones en las prácticas clínicas ha recibido, por tanto, una creciente atención en los años recientes. La doctrina legal del consentimiento informado -la cual requiere que el médico revele la naturaleza del procedimiento, las alternativas y los riesgos envueltos- se ha convertido en una conversación de "un sentido", sin que ello signifique la participación del paciente. Por lo tanto, hoy en día se hace necesario revalorizar el derecho de los pacientes a tener la oportunidad de ser participantes informados en las decisiones clínicas que atañen a su salud.

Diferentes países de Europa y Norteamérica han ido instalando esta temática en sus legislaciones y sistemas educativos. En Holanda, en 1995, el Parlamento promulgó una ley llamada “Acta de Contrato del Tratamiento Médico", que establece que los pacientes tienen el derecho a recibir información y los médicos y odontólogos tienen la obligación de informarles y pedir su autorización para los distintos procedimientos (consentimiento informado). La legislación mencionada estableció la responsabilidad de los pacientes en las decisiones sobre su salud y cuidado dental. Por otra parte, determina que los odontólogos deben comprometerse en invertir más tiempo para lograr que las decisiones sean formuladas libremente y en desarrollar los requisitos de las habilidades comunicacionales(3).

El odontólogo tiene un deber de conducta con su vida profesional, en concordancia con los principios éticos escritos en las reglas morales(4). Por lo tanto, además de garantizar la confidencialidad a sus pacientes, debe presentar las distintas alternativas de tratamiento, obtener su consentimiento y respetar sus decisiones(5).

En la práctica odontológica diaria el consentimiento informado se implementa como un documento en el cual se deja constancia de lo anteriormente descrito. Sin embargo, para que un paciente pueda firmar el consentimiento informado debe reunir determinadas condiciones, entre las que se cuentan: haber recibido y comprendido información suficiente, encontrarse libre y ser competente para tomar una decisión, a través de un documento que refuerce la situación contractual de la práctica moderna.

La concepción de consentimiento informado integra dos visiones, una jurídico-técnica y otra 
filosófica. Si el uso del mencionado documento se restringiera a atender solamente a la primera, significaría una poda de los alcances éticos del consentimiento, porque su correcto uso supone, también, el conocimiento de los fundamentos éticos en que basa su existencia. El fundamento del consentimiento informado es el ejercicio de la libertad de conciencia y, por ende, la elección responsable. El consentimiento informado es el proceso gradual, que se ubica en el seno de la relación odontólogo-paciente, en virtud del cual el paciente obtiene determinada información del profesional, en términos comprensibles, que le permite participar voluntaria, consciente y activamente en la adopción de decisiones respecto del tratamiento de su salud.

El uso del consentimiento informado permite trabajar en el contexto de una relación veraz. Al compartir la responsabilidad de la decisión, se logra el esfuerzo volitivo de los pacientes a favor de la ejecución del tratamiento ofrecido con la aceptación formal de los riesgos. El resultado final constituye un mejor servicio(6).

\section{El consentimiento informado en la formación universitaria}

La descripción anterior invita a reflexionar sobre la importancia que esta temática debiera tener en los currículos de las carreras y en los programas académicos relacionados con las ciencias de la salud.

Muchos de los planes de estudio universitarios fueron diseñados con un sentido local y regional ya superado, y no corresponden a la cosmovisión emergente. En cambio, vemos surgir programas de enseñanza transnacionales (Unión Europea), internacionales (bachilleratos, posgrados) o interregionales (MERCOSUR, Nafta, Asia Pacífico) ${ }^{1}$.

1 Pérez Lindo A. Mutaciones en los sistemas de ideas y cambio curricular. Conferencia dictada ante autoridades y personal docente de todas las unidades académicas de la U.N.N.E. Corrientes, 4 de mayo de 1995, Buenos Aires, Argentina.
Las recomendaciones del Consejo General Odontológico del Reino Unido dirigidas a la educación odontológica presentan un nuevo énfasis sobre la importancia de incluir la ética y las legislaciones respectivas en los currículos odontológicos. Asimismo, destacan que el deber de los estudiantes de odontología envuelve la protección de la vida y salud de sus pacientes, el respeto por su autonomía y la realización del consentimiento informado(7).

Un estudio sobre el impacto de la ética odontológica en el currículum, realizado en la Universidad de Minnesota(8), reveló que, luego de recibir 720 estudiantes un entrenamiento específico durante siete años, los beneficios alcanzados fueron altamente significativos.

Con todo, se presenta un nuevo desafío al ámbito universitario: tomar estos nuevos paradigmas reinterpretándolos, enriqueciéndolos o bien juzgándolos.

"(La universidad) ha sido una gran reproductora de visiones culturales y sociales, ha contribuido, como ninguna otra institución, a fijar las identidades de los actores sociales e individuales. Es lógico pensar entonces que la transformación de los modelos culturales tendrá un impacto significativo en el currículum universitario (...) ¿Es necesario tomar en cuenta estos contextos para el diseño curricular? ¿Con qué sistemas de ideas podemos establecer los parámetros epistemológicos, filosóficos, ideológicos, culturales y éticos que orienten la formación de los jóvenes? Son preguntas difíciles de responder. En todo caso, muestran que para acordar un cambio curricular se necesitan muchos diálogos, muchas confrontaciones, muchas reflexiones sobre los temas en cuestión. De lo contrario, podríamos llegar a formular una mera actualización funcional del currículum sin mucha consistencia teórica. Necesitamos una universidad-mundo, transcultural, interdisciplinaria, con fuerte base científica, 
productora de conocimientos, agente de cambios, pedagógicamente innovadora, ideológicamente clara en su función social. El modelo de una universidad así transformada brindará el marco necesario al cambio curricular ${ }^{2}$."

"En esa línea, el currículum universitario debe constituirse en este momento histórico, recuperando sus más genuinos propósitos e intereses académicos, para desarrollar una de las más complejas e importantes capacidades humanas: la capacidad de pensar (...) de manera inédita, osada, crítica, creativa y comprometida (...) Los derechos humanos tendrán que impactar al currículum universitario como contenidos específicos y como perspectiva general o enfoque (...) Actualmente, los nuevos lenguajes científicos y tecnológicos forman parte importante de las comunicaciones y las prácticas cotidianas en los distintos espacios sociales(9)".

Será necesario, entonces, dilucidar cómo el cambio del paradigma en las relaciones sanitarias debiera influir directamente sobre los diseños curriculares de carreras y programas académicos relacionados con las ciencias de la salud.

\section{Formación universitaria de posgrado}

Las Residencias Odontológicas Universitarias (ROU), de la Facultad de Odontología de la UNLP, son un sistema educativo del graduado reciente que tiene por objeto completar su formación integral ejercitándolo en el desempeño responsable y eficaz de la práctica odontológica. Se desarrollan según un programa educacional predeterminado, mediante la ejecución personal -bajo supervisión- de ac-

Pérez Lindo A. Mutaciones en los sistemas de ideas y cambio curricular. Conferencia dictada ante autoridades y personal docente de todas las unidades académicas de la U.N.N.E. Corrientes, 4 de mayo de 1995, Buenos Aires, Argentina. tos profesionales de complejidad y responsabilidad crecientes. Su objetivo fundamental es centrar la formación del odontólogo residente en el concepto de salud, pero no ya entendido como un asunto privado de cada persona, sino como una responsabilidad social que debe ser asumida con enfoque preventivo, mediante el mejoramiento de la salud de la población.

Se trata de mantener actitudes de apertura a las necesidades de salud bucodental actual o potencial y dar respuesta a las demandas sociales de tratamiento. La práctica está vinculada a la salud colectiva y a la resolución de los problemas concretos de la salud oral de la población. Por ello, las experiencias educativas propuestas a los residentes son pertinentes a estos objetivos. La formación es dependiente del proceso de prestación de servicios de salud, procurando articularse e integrarse con ellos, constituyendo así las metas importantes de la formación del odontólogo residente.

Al terminar el ciclo, los graduados han sido preparados para responder a un perfil profesional que comprende:

- Resolver la problemática de salud bucal correspondiente a los tres niveles de atención dentro de las incumbencias propias de la odontología general.

- Integrarse a cualquiera de los subsectores que componen la práctica profesional, en forma individual o en instituciones públicas y privadas del sector, contribuyendo con los objetivos de equidad, eficacia y eficiencia del sistema de atención en salud bucal.

Por todo lo anterior, las actividades de capacitación del odontólogo residente se basan en una amplia, comprometida y creciente participación en el trabajo comunitario de salud oral desde la realización de experiencias prácticas. 
Las ROU constituyen un sistema más, donde el graduado inscrito recibe una enseñanza permanente y continua, aplicada en la modalidad docencia-servicio con transferencia a la comunidad, para una mejor calidad de vida y salud bucal.

\section{El documento de consentimiento informado en la formación de posgrado}

La ausencia de una asignatura específicamente filosófica-que tenga como objetivos los tratados de la antropología filosófica y los fundamentos deontológicos del ejercicio profesional- en los diseños curriculares de las carreras de odontología y en el programa académico de posgrado de las ROU dificulta el aprendizaje integral de determinadas temáticas, entre otras: la libertad y dignidad humana o la conciencia moral, que pueden considerarse como contenidos previos y fundantes del consentimiento informado.

Esta falencia ha hecho que tales cuestiones queden subyacentes en otras áreas o asignaturas clínicas, como la Odontología Legal, y dependiendo del buen criterio de los docentes, quienes, por su estricta formación científica, carecen, en gran parte, de una formación filosófica. Sin embargo, se cree conveniente articular estos contenidos legales con los de naturaleza filosófica, de modo de presentar el tema del consentimiento informado con una perspectiva que integre los distintos enfoques pedagógicos.

Con todo, es prudente reforzar la formación académica ofrecida en las ROU con una que presente los contenidos de la bioética odontológica para facilitar la comprensión de los fundamentos éticos del consentimiento informado y enriquecer la cosmovisión integral del hombre.

En esta investigación, la hipótesis fue la siguiente: "La incorporación de una formación específica sobre aspectos bioéticos, jurídicos y odontológicos del denominado "documento de consentimiento informado" durante el programa académico de las Residencias Odontológicas Universitarias, favorecerá a un mejor conocimiento y desempeño clínico-profesional de los odontólogos residentes en el área de la odontología legal".

En virtud de lo anterior, se incorporó dicho espacio educativo en el programa de las ROU. De este modo, se realizó un estudio experimental controlado con dos grupos de odontólogos residentes pertenecientes al mismo programa. Cabe destacar que la presente investigación se encuentra en su primera etapa, que tuvo como objeto la medición preliminar de los grupos experimental y control antes de someter al primer grupo al espacio educativo mencionado.

\section{Objetivo}

Comparar datos obtenidos en relación con aspectos teóricos y clínicos de la práctica del consentimiento informado en profesionales odontólogos del Programa de Residencias Odontológicas Universitarias de la Facultad de Odontología de la UNLP.

\section{Materiales y métodos}

En el trabajo de investigación se tuvieron en cuenta dos variables.

a. La variable "conocimiento del proceso de consentimiento informado" en odontólogos pertenecientes a las ROU de la Facultad de Odontología de la UNLP.

b. La "práctica del proceso del consentimiento informado" en odontólogos pertenecientes a la ROU de la Facultad de Odontología de la UNLP.

Se efectuó un estudio descriptivo con 78 odontólogos, de ambos sexos, pertenecientes 
al Programa. De este modo, la recolección de datos se llevó a cabo en forma prospectiva. Se adoptó como criterio de exclusión a aquellos residentes que no estuvieron en las condiciones académicas y/o administrativas antes del 30 de abril de 2004; mientras que como criterio de eliminación la ausencia definitiva u otro motivo académico y/o administrativo que hubiese provocado la interrupción de su programa de Residencias Odontológicas Universitarias, como así también la incorporación de nuevos odontólogos admitidos como residentes en cada cohorte.

Se aplicó un primer instrumento, "Pre-test I", que buscó establecer un diagnóstico inicial de los grupos experimental y control. Éste indagó sobre el nivel de conocimiento de los contenidos propuestos: la identificación de conceptos de ética, moral, moralidad, conciencia, libertad; conceptos fundamentales de bioética odontológica; conceptos básicos en odontología legal, y los procedimientos legales para la ejecución del documento.

Se aplicó también un segundo instrumento de trabajo, "Pre-test II", que buscó ponderar la importancia del consentimiento informado en la práctica clínico-profesional de la odontología legal de la población objeto. Asimismo, se procuró obtener los siguientes datos: tiempo necesario para el cumplimiento del proceso de consentimiento informado; actividades de posgrado de formación; ofrecimiento de alternativas de tratamiento; respuesta del paciente; compromiso del paciente con el tratamiento; participación en la decisión del plan de tratamiento propuesto; importancia de su utilización para la actividad del odontólogo, e importancia de su utilización para el paciente.

Ambos instrumentos fueron estructurados, voluntarios y anónimos para salvaguardar la identidad de los sujetos experimentales. Asimismo, se sometieron a la evaluación de la
Asesoría Pedagógica de la Facultad de Odontología durante el primer trimestre del año.

Seguidamente, se realizó una formación académica específica, bajo la modalidad de seminario/taller, en lo que respecta al documento de consentimiento informado en el marco de la bioética odontológica. El seminario/taller estuvo estrechamente relacionado con la formación académica de Odontología Legal, que figura en el programa de las ROU durante el segundo cuatrimestre del ciclo lectivo 2004.

\section{Resultados y discusión}

Del análisis de los instrumentos aplicados (Pretest I y II) a 78 odontólogos, de ambos sexos, pertenecientes a las ROU, los resultados fueron:

De los encuestados, el 57,69\% de la población correspondió al sexo femenino; el rango de edades fue de 25 y 50 años; la edad media de 28,47 años; moda y mediana 28 años. El promedio de los años de graduados fue 3,5 años, mientras que la moda y mediana, 3 años.

En relación con la variable "aspectos teóricos de la práctica de consentimiento informado", sobre un total de 780 opciones totales, la población encuestada sólo contestó correctamente el 35,64\%.

La distribución en relación con el total de opciones (780) fue la siguiente: odontólogo género femenino: opciones correctas: 165 (21,15\%), opciones incorrectas: 285 (36,54\%); odontólogo género masculino: opciones correctas: $113(14,49 \%)$, opciones incorrectas: 217 $(27,82 \%)$. Del total de opciones correctas señaladas por los encuestados (278), 165 (59,35\%) correspondieron al género femenino, mientras que $113(40,65 \%)$ al género masculino.

Lo hallado en el Pre-test I reveló la necesidad de reforzar la formación académica de 
posgrado en relación con la temática. Países como Estados Unidos, España e Italia han generado no sólo jurisprudencia sino también numerosos espacios de formación académica. De 54 facultades de odontología de Estados Unidos que intervinieron en un estudio sobre la planificación de los planes de tratamiento, la mitad mostró tener incorporada la explicación de los riesgos del tratamiento y el pedido de consentimiento en el momento en que presentaban el plan propuesto, mientras que el resto de las unidades académicas lo hacía sólo posteriormente.

En relación con la variable "aspectos clínicos de la práctica de consentimiento informado", 51 (65,38\%) odontólogos contestaron afirmativamente que tenían un tiempo previsto en la consulta con su paciente para el diálogo y transmisión de información sobre el plan de tratamiento propuesto. De la tabla de contingencia se desprendió que, de aquellos que señalaron poseer un tiempo previsto, 29 (76,92\%) odontólogos correspondieron al género femenino. Además, se observó que fueron los más jóvenes y recientemente recibidos los que escogieron esta opción.

Ahora bien, 48 (61,54\%) residentes coincidieron en que dedicaban todo el tiempo necesario para esa actividad. De éstos, 29 (60,42\%) correspondieron al género femenino. En este caso también fueron los más jóvenes y recientemente graduados. No obstante, 24 (30,77\%) manifestaron dedicarle sólo diez minutos en su consulta odontológica al proceso de consentimiento informado, no existiendo en este caso diferencias entre géneros (12, 50\% masculino; $12,50 \%$ femenino). Todos los que eligieron esta última opción fueron jóvenes y recientemente graduados.

Algunas características de los profesionales pueden jugar un rol mayor en cómo se practica el consentimiento informado. Por ejemplo, las actitudes frente a malas prácticas o la previa exposición a una mala práctica parecen influir en el comportamiento. Profesionales que conocen bien a un paciente pueden llevar a cabo con él un proceso de consentimiento diferente al que realizan con uno más reciente; recíprocamente, los profesionales podrían creer que pueden predecir una respuesta del paciente basada en experiencias previas. A partir de estos ejemplos, se puede observar que el potencial de asociaciones entre las características de los profesionales y las de consentimientos prestados es todavía difícil de predecir. Además, éste tiene que ser estudiado todavía sistemáticamente(10).

Se requiere con urgencia el entrenamiento en las técnicas de consentimiento informado. La mentoría informal debe ser reconocida como un método de entrenamiento importante para el consentimiento informado, y ser respaldado por las autoridades para asegurar que los instructores de residentes lo practiquen efectivamente(11).

En la educación odontológica, los estudiantes invierten demasiado tiempo en los cuidados previstos para el tratamiento dental. En diversos programas académicos deben cumplir con requisitos que pueden dificultar la relación con sus pacientes. Probablemente, la solución a los conflictos que se presentan generalmente sea la utilización del consentimiento informado(12).

\section{Referencias}

1. Hartshorne JE. Principles of valid informed consent to treatment in dentistry. J Dent Assoc South Africa 1993; 48 (8): 465-468. 
El consentimiento informado en odontología - R. Miguel, M. Zemel

2. Manrique JL, Cottely E, Rossi RP, Smith R. Consentimiento informado: fundamento ético, médico y legal. Rev Argent Cirug 1991; 61: 99-102.

3. Eijkman MA. Dental health education: from education to informed decision making. Patient Educ Couns 2001; 42(2):101-104.

4. Nash DA. Ethics in dentistry: review and critique of Principles of Ethics and Code of Professional Conduct. J Am Assoc 1984; 109(4): 597-603.

5. Hirsch AC, et al. Ethics in dental practice. J Am Dent Assoc 1986; 113 (4): 559-603.

6. Secchi MA. Aspectos diversos de responsabilidad profesional. Rev Soc Argent Ped 1999; 15(2): 77-81.

7. Bridgman, et al. Teaching and assessing ethics and law in the dental curriculum. Br Dent $J$ 1999; 187(4): 217-219.

8. Bebeau MJ, et al. The impact of a dental ethics curriculum on moral reasoning. J Dent Educ 1994; 58(9): 684-692.

9. De Alba A. El currículum universitario ante los retos del siglo XXI, la paradoja entre posmodernidad, ausencia de utopía y determinación curricular. En: De Alba A, (coord.) El currículum universitario. De cara al nuevo milenio. México: Plaza y Valdés; 1997.

10. Braddock C, Micek M, Fryer-Edwards K, Levinson W. Factors that Predict Better Informed Consent. $J$ Clinical Ethics 2002; 13(4): 344-352.

11. McClean K, Card Sh. Informed consent skills in Internal Medicine Residency: How are Residents Taught, and what do they learn? Acad Med 2004; 79(2): 128-133.

12. Van Dam S. Requirement-driven dental education and the patient's right to informed consent. $J A m$ Coll Dent 2001; 68(3): 40-47.

Recibido: 28 de septiembre de 2005

Aceptado: 31 de enero de 2006 


\section{ANEXO}

Gráfico 1. Distribución por género

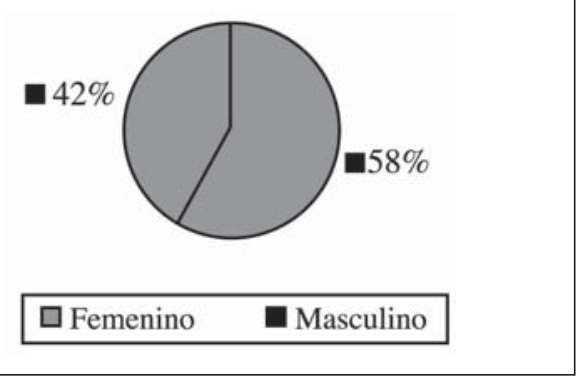

Gráfico 2. Evaluación de aspectos teóricos sobre el consentimiento informado en odontología.

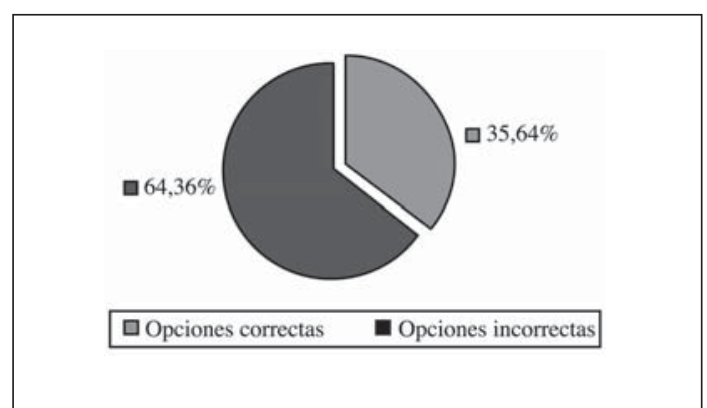

Gráfico 3. Espacio que posee el proceso de consentimiento informado en la consulta odontológica.

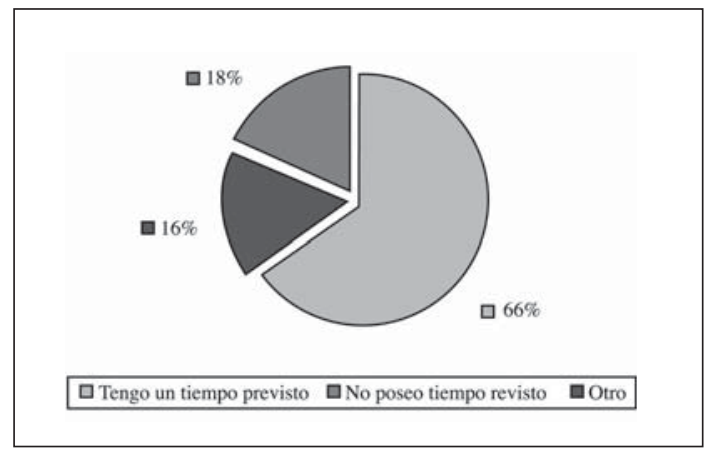

Gráfico 4. Tiempo que se utiliza habitualmente para el proceso de consentimiento informado en la consulta odontológica.

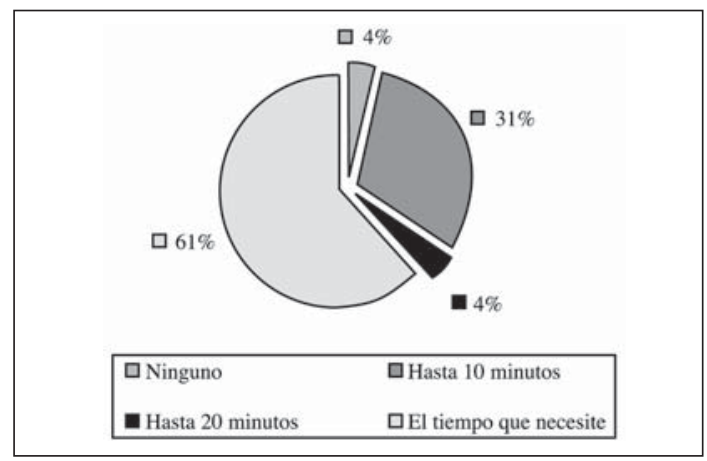

